\title{
INVESTIGACIÓN CUANTITATIVA (MONISMO METODOLÓGICO) Y CUALITATIVA (DUALISMO METODOLÓGICO): EL STATUS EPISTÉMICO DE LOS RESULTADOS DE LA INVESTIGACIÓN EN LAS DISCIPLINAS SOCIALES
}

\section{QUANTITATIVE RESEARCH (METHODOLOGICAL MONISM) AND QUALITATIVE RESEARCH (METHODOLOGICAL DUALISM): THE EPISTEMIC STATUS OF RESEARCH FINDINGS IN THE SOCIAL DISCIPLINES}

Dr. Héctor Salas (hsalas@fca.unam.mx) Facultad de Contaduría y Administración, Universidad Nacional Autónoma de México (México)

\begin{abstract}
This essay analyses the positions which are related to the quantitative and qualitative research models in the social disciplines; it examines the universality and invariance of scientific laws; and it presents the inadequacy of the conceptions of logical positivism and of classical epistemology, as well as the new concepts and two of the models which arose there from. Then, based on this it evaluates the epistemic status of research findings from both models, the scientific status of the disciplines and their theories and the rationality of the agreements reached over research conclusions: the limitations of scientific laws show the plausibility of quantitative research, which can be associated with the epistemic values of truth, intersubjectivity and susceptibility of replication, and its conclusions can be considered as genuine knowledge, though not those of qualitative research. But these philosophical notions leave open the controversy over the appropriate conceptions to explore the social phenomena.
\end{abstract}

Keywords: methodological monism, methodological dualism, knowledge, scientificness, rationality.

\section{Resumen}

Este ensayo analiza las posturas asociadas con los modelos cuantitativo y cualitativo de investigación en las disciplinas sociales; examina la universalidad e invariancia de las leyes científicas; y presenta las 
insuficiencias de las concepciones del positivismo lógico y de la epistemología clásica, así como los nuevos conceptos y dos modelos surgidos del mismo. A partir de esto, evalúa el status epistémico de los resultados de investigación bajo ambos modelos, el status de cientificidad de las disciplinas y teorías y la racionalidad de los consensos sobre las conclusiones de investigación: las limitaciones de las leyes científicas muestran como plausible la investigación cuantitativa, que puede asociarse a los valores epistémicos de verdad, intersubjetividad y replicabilidad, y sus conclusiones pueden considerarse como conocimiento genuino, pero no las de la investigación cualitativa. Pero estas propuestas filosóficas no permiten superar la controversia sobre las concepciones y enfoques apropiados para explorar los fenómenos sociales.

Palabras clave: monismo metodológico, dualismo metodológico, conocimiento, cientificidad, racionalidad.

\section{Introducción}

Los métodos cuantitativos o cualitativos empleados en investigación en las disciplinas sociales corresponden respectivamente a las posturas epistemológicas del monismo metodológico (que propone aplicar a las disciplinas sociales los modelos de la filosofía de las ciencias naturales) y del dualismo metodológico (que sostiene que en las disciplinas sociales deben aplicarse modelos específicos para las mismas). Estas posturas circunscriben enfoques y diseños de investigación bajo los que se llevan a cabo los proyectos, determinándose así el tipo de resultados a obtener, así como la índole de las conclusiones a establecer. Ambos enfoques parten de la forma en que el investigador concibe la naturaleza de los hechos y los fenómenos sociales, adicionalmente a las particularidades que privan en su especialidad. Esto determina los objetivos y metodologías que adoptará para la investigación, su actitud hacia los valores epistémicos, y los modelos del conocimiento, cientificidad y racionalidad que buscará seguir. Pero hay una correspondencia sólo parcial con estos últimos conceptos y modelos, y debe acotarse su aplicación en el ámbito de la investigación en las disciplinas sociales; al mismo tiempo que los resultados de ambos modelos de investigación tienen distinta condición frente a dos concepciones del conocimiento.

El propósito de este ensayo (1) es plantear algunas consideraciones sobre el status epistémico de los resultados y conclusiones que producen ambos modelos de investigación en las disciplinas sociales, sobre el status de cientificidad de las disciplinas y sus teorías y sobre la racionalidad de los consensos alcanzados en torno a las conclusiones de la investigación y sus implicaciones para la teoría. Con esta finalidad se destacan primeramente algunos factores involucrados en la elección de un modelo de investigación por parte del investigador, y se analizan elementos epistemológicos y de la filosofía de la ciencia tales como los valores epistémicos, los modelos del conocimiento y las leyes científicas y las generalizaciones empíricas. Se analizan también los criterios de cientificidad y racionalidad que proponen dos modelos filosóficos vigentes -los programas de investigación científica de Imre Lakatos y el constructivismo pluralista de León Olivé-, y que son consistentes el primero de ellos con la investigación en las ciencias naturales, y el segundo con la investigación en éstas y en las disciplinas sociales -específicamente bajo el modelo cuantitativo. Asimismo, se describe la ruptura del consenso en torno al positivismo lógico, que originó el planteamiento de nuevos modelos en filosofía de la ciencia y la reformulación de los conceptos y el modelo epistemológico clásicos.

Este texto se divide en una sección que presenta las visiones de la naturaleza y de la naturaleza humana, los objetivos de la investigación y las metodologías correspondientes a las posturas del monismo y dualismo metodológicos. La segunda sección, en el contexto de la finalidad de establecer el conocimiento mediante la investigación en las disciplinas sociales, analiza dos puntos de vista sobre el mismo contenidos en los conceptos de saber y de conocer, y perfila las concepciones clásicas del conocimiento y de los valores 
epistémicos que lo caracterizan. La tercera sección analiza algunas limitaciones a la noción tradicional de las leyes científicas; y la cuarta detalla la crisis en la filosofía del positivismo lógico y el justificacionismo. La quinta sección delinea dos modelos filosóficos como algunos de los nuevos caminos a partir de dicha crisis: los programas de investigacióncientífica de Imre Lakatos, en el pensamiento del convencionalismo y más allá de la visión del justificacionismo, y consistente con la naturaleza del monismo metodológico; y el constructivismo pluralista de León Olivé que busca legitimar el pluralismo teórico a partir de una epistemología reformulada. Y en la última sección se analiza el status de ambas posturas metodológicas en relación con los planteamientos anteriores. Finalmente, se presentan algunas consideraciones adicionales y conclusiones.

\section{El monismo y el dualismo metodológicos: dos concepciones de los fenómenos humanos}

En los ámbitos de investigación de las disciplinas sociales, al igual que en filosofía de la ciencia, prevalecen dos puntos de vista con respecto de la forma en que se estima factible abordar la exploración y el conocimiento de los hechos y los fenómenos sociales en cuanto a los objetivos, metodologías y criterios de validez de sus resultados y conclusiones, entre otros aspectos que se considera que deben caracterizar a los estudios correspondientes. De acuerdo con los planteamientos del naturalismo o monismo metodológico, mismo que se asocia con el punto de vista positivista, los modelos de la filosofía de las ciencias naturales deben aplicarse también en la investigación en las disciplinas sociales; mientras que bajo la propuesta del dualismo metodológico, consistente con enfoques constructivistas y pluralistas de las teorías sociales, se sostiene que deben ser modelos de cientificidad y racionalidad específicos para las mismas los que orienten la investigación bajo métodos propios, y establezcan los criterios de justificación de sus resultados (Velasco 1995a, 1995b, 2000).

En las disciplinas sociales, quienes se adhieren a las tesis del monismo metodológico realizan su investigación en especialidades donde se han desarrollado metodologías convencionales (2) para definir y cuantificar las variables que intervienen en los eventos sociales, quedando así registrados de manera intersubjetiva hechos observables -pero ámbitos como los objetos de estudio del conductismo, de la econometría o de las finanzas corporativas resultan estrechos en el contexto total de cada disciplina. Estas comunidades de investigación adoptan las directrices positivistas de evitar la subjetividad y presentar opiniones expertas como el producto de una investigación; así, sus proyectos replicables por otros investigadores llevan a cabo pruebas de hipótesis con modelos causales mediante análisis cuantitativos con rigor metodológico, con el objetivo de explicar las regularidades que se disciernen en los fenómenos, estableciendo con confianza estadística sus determinantes. Los resultados se generalizan como hechos a universos de referencia desde muestras representativas, para sentar conclusiones e implicaciones para la teoría que se muestren como conocimiento válido intersubjetivamente para todo investigador; y la intersubjetividad de modelos y decisiones metodológicas busca ser trascendida al relajarse los supuestos originales en análisis subsiguientes y obtenerse resultados consistentes.

Por su parte, los proponentes del dualismo metodológico consideran viable sólo la descripción, comprensión e interpretación personal y casuística de fenómenos sociales que son percibidos como ambiguos, inaprensibles e inacabados; afirmando que los mismos pueden entenderse únicamente mediante métodos basados en una visión global de múltiples elementos con relaciones de complementariedad en sistemas complejos de conducta incierta. Estos grupos sostienen que en la naturaleza pueden encontrarse regularidades y establecerse leyes universales; pero que en los eventos de la naturaleza humana, donde 
interactúan numerosas variables interdependientes de difícil definición, medición y observación, la pretensión de discernir regularidades difusas y con causalidad probabilística y multidireccional, así como con efectos multiplicativos no lineales, surge de un enfoque desde la simplicidad, que postula la aplicación de metodologías que corresponden a una concepción mecanicista y reduccionista del mundo (Etkin y Schvarstein 1995, Morin 1996, Etkin 1996).

Así, en los amplios espacios de las disciplinas sociales en cuyos fenómenos no se disciernen variables empíricas intersubjetivamente definibles o cuantificables, la investigación cualitativa se traza el objetivo de realizar estudios de caso descriptivos con conclusiones casuísticas no generalizables basadas en juicios $e$ inferencias personales que parten de la comprensión e interpretación de eventos sociales complejos bajo sofisticados marcos conceptuales y modelos analíticos. Asimismo, con frecuencia, parte o toda la evidencia que se analiza cualitativamente son las opiniones y apreciaciones personales de los integrantes de alguna muestra obtenidos mediante entrevistas o cuestionarios. Por tanto, esta línea de pensamiento acepta como cierto e inevitable el que dos investigadores que trabajaran sobre un mismo objeto de estudio y en un mismo momento, con los mismos objetivos del proyecto de investigación y bajo el mismo método cualitativo, no necesariamente llegarían a las mismas conclusiones.

Este enfoque se encuentra en proceso de ser conformado como un modelo de investigación, y no ha alcanzado aún el nivel de estructuración del pensamiento positivista ni proporciona el mismo nivel de comprensión (Rendón y Montaño 2004). De hecho, el propósito de aprehender los fenómenos de la naturaleza humana en toda su riqueza y profundidad se encuentra permeado de un fuerte componente subjetivo, y se caracteriza en la práctica de investigación por juicios y opiniones expertas; siendo discutible si existen allí verdaderas comunidades de investigación donde se consensuen diseños, supuestos, características y desarrollo de los proyectos de investigación, y cuyos resultados y conclusiones tengan validez intersubjetiva para todos sus integrantes.

En sí, la controversia entre ambos modelos de investigación gira en torno a la concepción por parte del investigador de la naturaleza de los hechos y los fenómenos sociales -e inciden también en ella destacadamente características particulares de algunas de las especialidades, como por ejemplo, el tipo de variables que son características en las áreas cuantitativas de las disciplinas económico administrativas. Consecuentemente a partir de esto, se determinan objetivos y diseños de investigación, la concepción del conocimiento y la actitud hacia los valores epistémicos, así como los modelos de cientificidad y racionalidad que se adoptarán.

\section{La visión clásica de saber o del conocimiento y de los valores epistémicos}

En relación con los resultados de la investigación, ambas posturas metodológicas parten de concepciones distintas del conocimiento -ya sea bajo el concepto de saber o bajo el de conocer- y de una diferente actitud hacia las virtudes epistémicas -sobre todo la verdad, la certeza, la objetividad, la replicabilidad y la precisión (Daston y Galison 2007).

\subsection{Saber y conocer}

El propósito de hacer una aportación al conocimiento mediante la investigación desde ambos modelos se dirige hacia dos concepciones del mismo representadas en dos conceptos distintos: saber y conocer. Para conocer algo es preciso tener una experiencia personal y directa; estar familiarizado. Conocer no consiste en 
un solo acto, sino en muchas experiencias variadas, capaces de ser integradas en una unidad; por ello el conocimiento puede ser más o menos complejo, más o menos rico. En su sentido más rico, conocer implica poder contestar múltiples y variadas cuestiones de la más diversa índole sobre un objeto o un tema; pero saber muchas cosas sobre él implica una relación cognoscitiva diferente a la de quien realmente lo conoce. Conocer no es una suma de saberes, sino una fuente de ellos; el saber es necesariamente parcial, el conocer aspira a captar una totalidad. Saber y conocer no se justifican de la misma manera: el saber se justifica en razones objetivamente suficientes, comprobables por cualquiera que tenga acceso a ellas; el conocer se justifica en experiencias directas que son personales e intransmisibles (Villoro 1982).

El que el saber se justifique mediante razones transmisibles y comprobables por todos permite alcanzar consensos en el seno de las comunidades de investigación; el saber se encuentra en el contexto de la intersubjetividad. El conocer, justificándose en experiencias intransmisibles, y no en hechos intersubjetivos, se encuentra en el contexto de la subjetividad; y bajo este esquema, el valor de ideas y planteamientos -que se encuentran propiamente en el ámbito de la opinión- frecuentemente está asociado con la autoridad y prestigio personales del investigador.

\subsection{Las nociones clásicas}

La noción clásica del conocimiento como creencia verdadera y justificada traza su origen al análisis tripartito de Platón, quien lo definió como una creencia que es verdadera, y de la cual la persona cuenta con una explicación racional o justificación. Estas tres condiciones -el que una idea sea aceptada por una persona como creencia, el que esa idea sea verdadera, y el que esa persona pueda justificar o fundamentar su creencia- son las condiciones necesarias y suficientes de la descripción usual del conocimiento o de saber (Nozick 2008, Sellars 2008, Pojman 2001). Otras nociones clásicas son:

a) La verdad y la certeza

La verdad consiste en una correspondencia entre las proposiciones o los juicios y la realidad (Olivé 2000), es decir, una idea es verdadera si corresponde fielmente al fenómeno que representa. Ésta es la teoría clásica de la verdad o teoría de la correspondencia (Popper 1979) (3). Establecer como conocimiento leyes y teorías mediante la corroboración de su verdad contra la evidencia empírica representa uno de los propósitos de la investigación científica. Así, durante las primeras décadas del siglo pasado el positivismo lógico del Círculo de Viena adoptó un criterio de cientificidad, o de demarcación entre ciencia y no-ciencia, de acuerdo con el cual proposiciones y teorías son científicas en tanto pueda verificarse o justificarse su verdad mediante la contrastación de las mismas con la experiencia. Este criterio permitía disociar lo científico de lo metafísico, y distinguir entre conocimiento y opinión.

Asimismo, su criterio de verdad para enunciados, leyes y teorías consistía en definir para su verificación empírica las condiciones bajo las cuales era verdadero o falso lo que afirmaban (Carnap 1961). Para el positivismo lógico, científico era lo verificable, y verdadero, lo verificado. La verdad de enunciados, leyes o teorías que hubiera sido verificada por la investigación científica había sido establecida, y había sido establecida con certeza, al referirse estas nociones a la verdad absoluta y al conocimiento infalible -y "la honestidad científica exigía que nada afirmara uno que no hubiera sido probado" (Lakatos 1970:94). 
b) La objetividad y la replicabilidad

En uno de los sentidos de objetivo, que se asume en toda la tradición platónica, una creencia es objetiva porque se refiere a un hecho objetivo, cuya existencia en nada depende de algún sujeto, ni individual ni colectivo, ni está dada intersubjetivamente (Olivé 2000). Al investigar los hechos, la objetividad radica en metodologías que eliminen la subjetividad del investigador. La ciencia busca aprehender las regularidades que exhibe la naturaleza, como las constantes universales y las leyes que asocian necesariamente a un efecto con sus causas, al establecer la verdad de las hipótesis bajo metodologías rigurosas que prescindan de inferencias y juicios personales; al capturar y registrar datos sin distorsiones por sesgos del investigador; y al definir y cuantificar variables e indicadores con metodologías de carácter convencional, consensuadas en las comunidades de investigación (Daston y Galison 2007).

Esto ocurre también en la investigación en las áreas cuantitativas de las disciplinas sociales; y al registrarse hechos mediante metodologías aceptadas por consenso como una convención de las comunidades de investigación, esos hechos adquieren carácter y validez intersubjetivos, y las consecuencias de la contrastación de hipótesis pueden ser generalizadas a universos de referencia desde muestras representativas, quedando establecidas también como situaciones de hecho válidas intersubjetivamente para tales comunidades. Asimismo, al replicarse un estudio con nuevos supuestos, decisiones metodológicas, muestras y/o períodos, busca trascenderse la intersubjetividad de sus conclusiones, al obtenerse resultados consistentes con los originales y autentificarse las relaciones causales establecidas obviándose connotaciones de relativismo.

c) La precisión

Una buena teoría científica debe ser precisa, en tanto que sus predicciones, o las consecuencias deducibles de ella, deben concordar con los hechos - permitiendo su corroboración empírica. Entre las cualidades que debe poseer una teoría, la que muestra casi siempre ser decisiva para preferir una teoría sobre otra es la precisión; y aunque ésta debe incluir la concordancia cuantitativa y cualitativa entre sus predicciones y las observaciones existentes, crecientemente ha llegado a denotar básicamente concordancia cuantitativa (Kuhn 1977) Thomas Kuhn destaca también el papel de la precisión en las revoluciones científicas: "La ciencia normal conduce a un detalle de la información y a una precisión en la correspondencia de la teoría con la observación [que] tienen un valor que trasciende su generalmente no muy alto interés intrínseco. [Porque] la novedad ordinariamente surge sólo para quien, sabiendo con precisión lo que debería esperar, es capaz de reconocer que algo está mal. La anomalía aparece sólo contra el fondo que proporciona el paradigma. Mientras más preciso y de mayor alcance es el paradigma, es más sensible como indicador de una anomalía, y por lo tanto de una ocasión para el cambio de paradigma" (1964:65) (4).

Ciertamente, tales anomalías pueden observarse sólo en teorías altamente desarrolladas en campos que han llegado a su madurez; lo cual muestra el nivel de precisión en la descripción y explicación de sus eventos que han podido alcanzar ahora las ciencias de la naturaleza.

Pero eventualmente los conceptos clásicos de conocimiento (como conocimiento probado, infalible), verdad (como verdad absoluta) y objetividad mostraron no corresponder a los patrones de desarrollo de la ciencia, debiendo ser reformulados. 


\section{Limitaciones de la concepción tradicional de las leyes científicas}

Frecuentemente se considera como el propósito central de la investigación científica discernir y establecer las leyes que describen y explican el comportamiento de los fenómenos que se observan en el entorno, entendidas como generalizaciones sustanciadas empíricamente de las regularidades con que se manifiestan dichos fenómenos (Carnap, Popper, Hempel y Nagel, entre otros, afirman que el objetivo de la ciencia es elaborar leyes y teorías). Se considera a tales leyes como las unidades básicas del conocimiento científico, y típicamente son presentadas como invariantes y de aplicación universal; siendo precisamente su universalidad e invariancia lo que les da su valor y autoridad como generalizaciones (Allchin 2007). Sin embargo, es pertinente destacar que en las ciencias naturales las leyes no son invariantes ni de aplicación universal, y que las regularidades pueden observarse solamente en ámbitos limitados a ciertos rangos, quedando también su invariancia sujeta a condiciones.

Exceptuando la física, las leyes son comparativamente triviales, por ejemplo, en el caso de la química, donde todavía no pueden aplicarse plenamente buenos modelos matemáticos. En química, la verdadera teoría sería teoría física; y lo que experimento y razonamiento químicos pueden producir en su lugar son generalizaciones empíricas mucho menos fundamentales que las leyes de la física (Knight 1992). En este contexto, la ley de Boyle, que describe la regularidad que asocia cambios en presión y volumen de un gas a temperatura constante, y que representa un ícono cultural que epitoma el concepto de ley científica, no es universal ni invariante, tal como lo implica el término ley; y como muchas otras, puede considerarse como una ley sólo cuando es acotada en cierto contexto -el cual está dado por el tipo de gases y por cierto volumen mínimo especificado, entre otras condiciones. Ciertamente, esto muestra una visión más compleja de una naturaleza que exhibe sólo ámbitos limitados de regularidad o regularidades sujetas a rangos determinados; y únicamente dentro de los cuales se observa el fenómeno en los términos que predice la ley. Esto es, el ámbito circunscribe la universalidad, y las condiciones límite y las excepciones restringen la invariancia -el contexto es clave (Allchin 2007).

Asimismo, "toda generalización biológica parece admitir excepciones. Aparentemente, incluso la Ley de la Segregación de Mendel no es universal" y en consecuencia, la mayoría de los biólogos y "filósofos de la ciencia han optado por una comprensión de la biología como una aplicación casuística de modelos abstractos, más que como una aplicación sistemática de leyes universales" (Waters 1998:5). Y también se observan limitaciones en leyes de física como las leyes de la dinámica de Newton, que no aplican a velocidades relativistas; la ley del péndulo de Galileo, que debe matizarse con supuestos y condiciones límite que más bien representan idealizaciones irrealizables; la ley de resistencia eléctrica de Ohm, que acepta numerosas excepciones; o la ley de refracción de Snel (Allchin 2007).

El énfasis es en el contexto: científicos y filósofos de la ciencia consideran a las leyes como contextuales en última instancia. Para Stephen Toulmin (1960), las leyes tienen un dominio o ámbito particular de aplicación; y, como Kuhn (1964), sugiere que la articulación de tal ámbito es una de las tareas principales de la investigación científica. Pero entonces es sólo eliminando las condiciones limitantes que una ley adquiere la ilusión de universalidad, y alcanza su status sólo por la especificación arbitraria de condiciones generales control que es informativo localmente, pero no válido universalmente. Las leyes son locales, no universales, y las generalidades son contingentes, no invariantes (Allchin 2007).

No obstante, las filosofías de la ciencia tradicionales han sostenido "que las leyes universales juegan un papel central en el conocimiento científico [así que, aun cuando] numerosos aspectos de las filosofías 
tradicionales han sido correctamente rechazados, muchos filósofos y [científicos] estarían de acuerdo en que la comprensión científica requiere de enunciados generales de naturaleza empírica a pesar de que tales enunciados no satisfagan todos los criterios tradicionalmente atribuidos a 'las leyes'” (Waters 1998:5).

Por otra parte, las leyes universales no son las únicas. Ambrosio Velasco añade: “Existen también leyes estadísticas o probabilísticas [pero] mientras que Carnap, Hempel, Rescher, Salmon y otros autores las aceptan como leyes, Popper niega que sean leyes porque no son enunciados científicos. Para él todo enunciado científico debe ser refutable empíricamente, y los enunciados probabilísticos no lo son de manera estricta y concluyente (aunque desde luego son empíricamente corregibles). Sin embargo, el uso de leyes estadísticas incluso en teorías de ciencias maduras (mecánica cuántica) constituye un argumento inobjetable para su aceptación y legitimidad por parte de los filósofos. Aún más, en algunas teorías, como en la mecánica cuántica, las leyes estadísticas no son reductibles a leyes deterministas o universales, y no por ello deben considerarse como leyes de rango epistémico menor a las universales" (Velasco 2000:32).

Este indeterminismo ciertamente caracteriza a los fenómenos y eventos sociales, observándose relaciones causales de índole probabilística entre sus variables; lo cual, no obstante, permite a los profesionales de diversas formaciones en las disciplinas sociales intervenir para inducir y conducir el cambio y moldear el entorno social, así como el comportamiento de individuos, grupos, organizaciones o sociedades.

\section{La crisis del positivismo y del justificacionismo}

La crisis del positivismo lógico se relaciona con eventos que tuvieron lugar en física con "las sorprendentemente precisas leyes de la Mecánica de Newton," que siendo la teoría científica mejor verificada de todos los tiempos representaba una teoría verdadera -y con la certeza del conocimiento infalible. Así, a fines del siglo XIX, "se hicieron patentes ciertas desviaciones de dichas leyes; y aunque éstas eran pequeñas, eran de una naturaleza tan fundamental que todo el edificio del universo mecánico de Newton comenzó a derrumbarse" (Barnett 1974:15).

Paralelamente, en el ámbito de la filosofía de la ciencia se impugna la justificación lógica del método inductivo empirista, ya que una serie finita de observaciones no puede establecer con certeza la verdad de enunciados universales tales como las leyes, que están destinadas a abarcar un número infinito de casos. Esto y la crisis en la física clásica dejan sin sustento a la verificación como criterio de cientificidad del positivismo y a su criterio de verdad(Ayer 1956, Lakatos 1970, Hempel 1965, Schlick 1965).

Rudolf Carnap y otros justificacionistasproponen entonces adecuar el verificacionismo a un probabilismo, que destacara teorías y leyes altamente probables como las de la mecánica de Newton -el justificacionismo identifica el conocimiento con el conocimiento probado, y fue la tradición dominante en el pensamiento racional durante siglos; se sustituye inicialmente el verificacionismo del positivismo lógico por el probabilismo o neojustificacionismo, con el criterio de cientificidad probabilístico de confirmabilidad empírica. Pero Karl Popper observa que toda ley y teoría tienen una probabilidad de cero, al referirse muestras de cualquier tamaño a un universo infinito, y plantea la falsación de teorías, con un criterio de cientificidad para una teoría consistente en que ésta genere predicciones falsables; y de la cual posteriormente se señalan también sus insuficiencias -la versión dogmática del falsacionismo constituye la forma más débil del justificacionismo, con una noción de corroboración empírica equivalente a la de confirmación, pero que no representa el criterio de demarcación (Lakatos 1970, Velasco 2000). 
Más adelante, Kuhn cuestiona la racionalidad en la ciencia mostrando que se aceptan provisionalmente teorías que no sólo no han sido probadas, sino que no pueden serlo; y las cuales son contrastadas contra una evidencia empírica de carácter convencionalista y falibilista, es decir, carente de certeza y objetividad al tener que ser inevitablemente interpretada y evaluada intersubjetivamente en el seno de las comunidades científicas (5). Las teorías no se aceptan ni rechazan por comparación directa con la naturaleza; aunque esto no significa que experiencia y experimento no sean esenciales en el proceso en el que los científicos las rechazan (Kuhn1964). Las mismas son aceptadas o rechazadas en procesos de decisión extra-racionales basados en acuerdos o convenciones consensuados en dichas comunidades -convencionalismo que sería objeto de estudio de la sicología social más que de la filosofía de la ciencia, como sugiere Kuhn (6).

Ver que no es posible probar el conocimiento ni asignársele probabilidades es, dice Bertrand Russell, abandonar casi todo lo que se considera como conocimiento por la ciencia y el sentido común. El problema de la inducción retrae el pensamiento al falsacionismo; y buscando salvar la racionalidad en la ciencia, Popper plantea una teoría del conocimiento por prueba y error, por conjetura y refutación, resaltando la asimetría lógica de que mientras un ejemplo contrario refuta formalmente una ley, ningún número de observaciones la verifica formalmente.

Popper (1979) concibe teorías y leyes como conjeturas: supuestos sobre el mundo no inferidos por inducción de enunciados observacionales, que no son verificables, y de los que quizá nunca sepamos si son verdaderos, pero que pueden someterse a intentos por refutarlos -aquí Kuhn coincide en que quizá "hemos de renunciar a la noción explícita o implícita de que los cambios de paradigmas [nos llevan] más y más cerca de la verdad" (Kuhn 1964:169).

Finalmente, con respecto al falsacionismo, Alfred Ayer (1956) señala que es tan poco factible verificar como refutar concluyentemente una hipótesis; pues al observarse un hecho que la falsara, nunca habría la certeza de que habían permanecido invariables todas las condiciones pertinentes, y no se sabría si en realidad la hipótesis aún permanecía vigente. Imre Lakatos (1970) concluye que las teorías deben ser interpretadas normalmente como conteniendo una cláusula ceteris paribus, pero dado el falibilismo humano nunca podría establecerse si ha sido refutada una teoría junto con esta cláusula -y Popper (1969) admite que nunca puede refutarse concluyentemente una teoría (7).

Así, no pueden ser probadas ni probabilificadas ni refutadas formalmente teorías y leyes; las cuales, de haber representado conocimiento probado y conocimiento probable, constituyen de hecho conjeturas falsables (8).

De esta manera se muestran inadecuadas para describir y explicar el desarrollo, el progreso y la racionalidad en la ciencia las nociones clásicas, al igual que el pensamiento justificacionista.

\section{La ruptura con el justificacionismo y la búsqueda de nuevos caminos}

a) Una propuesta desde el monismo metodológico: el convencionalismo de Imre Lakatos

Poco después de ocurrir la crisis en la física, la visión de Einstein, libre de los supuestos de Newton, y cambiando fundamentalmente las ideas no cuestionadas desde tiempo inmemorial, pudo dar cuenta con precisión de los fenómenos (Russell 1969).Pero aunque su obra reconstruyó la explicación teórica en la disciplina, Lakatos señala que: "Ahora pocos filósofos o científicos piensan aún que el conocimiento 
científico es, o puede ser, conocimiento probado. Pero pocos se dan cuenta de que con esto toda la estructura clásica de los valores intelectuales queda en ruinas y debe ser reemplazada: uno no puede simplemente diluir el ideal de la verdad probada -como lo hacen algunos empiristas lógicos- al ideal de 'verdad probable' o -como lo hacen algunos sociólogos del conocimiento- a 'la verdad por consenso [cambiante]'” (Lakatos 1970:92). Como ocurre en los cambios de paradigmas en las revoluciones científicas.

A partir de tal situación, Lakatos (1970) busca restituir la racionalidad y un criterio de demarcación a la ciencia afirmando primeramente que en realidad Kuhn cuestiona una interpretación ingenua del falsacionismo, y que la postura de Popper (1969) implica un falsacionismo metodológico sofisticado, como una forma de convencionalismo(9), que ubica a las teorías en los contextos más amplios de programas de investigacióncientífica(10).Lakatos desarrolla entonces la metodología de los programas trazando reglas sobre caminos a evitar y caminos a seguir en investigación (es decir, heurísticas negativa y positiva, respectivamente), con relación a un núcleo teórico básico del programa -que es irrefutable por acuerdo entre los científicos- y a un cinturón protector de hipótesis auxiliares; y plantea también cinco tipos de decisiones metodológicas que son consensuadas en una comunidad científica.

La racionalidad en la actividad científica desde tales contextos amplios se encuentra en que en la sustitución de una teoría por otra dentro de un programa de investigación haya un progreso teórico, al explicarse todos los hechos no refutados de la teoría anterior, y predecirse nuevos hechos; y un progreso empírico, al corroborarse esas nuevas predicciones. Entonces, en un programa de investigación, el criterio de cientificidad consiste en que cada nueva teoríaen unaserie de teorías falsables tenga contenido empírico excedente corroborado sobre la anterior -es decir, progreso teórico y empírico-y el criterio de racionalidad consiste en que la retención o sustitución de teorías en el programa continúe produciendo progreso empírico. En palabras de Lakatos: "La racionalidad opera más lentamente de lo que piensa la mayoría, e incluso entonces, faliblemente" (1970:174). Y los mismos criterios se aplican en la sustitución de un programa de investigación por otro.

Finalmente, Lakatos (1970) plantea el requerimiento de un continuo crecimiento de la ciencia y un criterio de demarcación entre ciencia madura y ciencia inmadura: aquélla tiene poder heurístico y consiste de programas de investigación que anticipan no sólo nuevos hechos sino también nuevas teorías auxiliares; mientras que la segunda constituye un simple patrón de prueba y error -todo lo cual corresponde a la situación prevaleciente en la etapa pre-paradigmática, ya descrita por Kuhn como una etapa pre-científica en la que los campos del conocimiento aún no han madurado como ciencias.

\section{b) Una propuesta desde el dualismo metodológico: el constructivismo pluralista (11)}

Los patrones de desarrollo de la ciencia perfilados por distintos autores desde el siglo XIX (12) encierran problemas epistemológicos como el de la inconmensurabilidad entre paradigmas (13). Dos visiones del mundo son inconmensurables en el nivel epistemológico si no hay criterios comunes para decidir cuáles creencias son las correctas (Olivé 1999). Ludwick Fleck (1986) explica que los hechos científicos tienen una génesis y desarrollo, y no existen independientemente de las personas; son construcciones sociales. La tesis del constructivismo es que los marcos conceptuales tienen una fuerte implicación ontológica: son constitutivos de los objetos de conocimiento (Boyd 1992). El contenido de las teorías científicas está determinado por el paradigma que comparten los miembros de una comunidad. 
Un marco conceptual o paradigma contiene supuestos metafísicos, valores epistemológicos y éticos, normas metodológicas y otros elementos, y es compartido por los miembros de una comunidad epistémica o científica, permitiéndoles comprender y discutir racionalmente sus ideas, y lograr consensos racionales falibles de que ciertas proposiciones son razones que justifican a otras proposiciones (Olivé 1999, 2000).

Hilary Putnam afirma que "los 'objetos' no existen independientemente de los esquemas conceptuales. Nosotros recortamos el mundo en objetos cuando introducimos algún esquema de descripción" (1981:52). Los esquemas conceptuales, entonces, no son meros intermediarios entre los sujetos y los objetos, son más bien una pieza clave en la construcción de los objetos. "Los 'objetos' mismos son tanto algo que se hace como algo que se descubre, tanto productos de nuestra invención conceptual como del factor 'objetivo' de la experiencia, el factor independiente de nuestra voluntad" (1981:54).

Esto da lugar al fenómeno de la relatividad conceptual, que "depende del hecho de que [...] las nociones de objeto y existencia tienen una multitud de usos diferentes, y no un 'significado' absoluto" (Putnam 1987:19) y "trae consigo un pluralismo ontológico, pues abre la posibilidad de tener concepciones del mundo con ontologías distintas -incluso incompatibles- que sean igualmente adecuadas en ciertos contextos, en función de determinados intereses y objetivos" (Pérez 1999:210) (14).

León Olivé (1999) plantea que el constructivismo comparte la tesis central realista sobre la existencia de una realidad estructurada con independencia de todo marco conceptual, pero no la del realismo metafísico de que existe una única descripción completa y verdadera de ella. Esta idea se basa en las concepciones clásicas tanto del conocimiento como de la verdad como correspondencia entre las ideas y la realidad; y ya que la realidad sería accesible infaliblemente sólo desde el punto de vista del ojo de Dios, se desemboca en el escepticismo, pues nunca sabremos si tenemos un conocimiento genuino del mundo -lo cual está en línea con una concepción de teorías y leyes científicas como conjeturas falsables.

Pero en realidad, toda interpretación parte necesariamente de algún punto de vista; y como no hay razones para creer que todas ellas deban converger en una única representación, se conduce a un pluralismo teórico, pero en el que bajo la epistemología clásica cuando más una de esas teorías sería verdadera. Sin embargo, para el constructivismo distintas interpretaciones del mundo pueden ser correctas y constituir conocimiento genuino, tales como los paradigmas que han estado vigentes en distintos momentos a lo largo de la historia. Así, una concepción alternativa de saber bajo una epistemología reformulada sin la condición de verdad absoluta, permite tener un conocimiento genuino de la realidad como un saber falible.

Para Luis Villoro "el análisis tradicional de 'saber', al incluir la verdad absoluta de lo sabido, nos da una definición de ese concepto, que no es falsa sino inaplicable [así] sólo sabríamos las proposiciones infalibles" (1982:183). De hecho, "puedo saber algo sin tener que rechazar de antemano cualquier razón posterior en contra [...] Si saber implica la verdad absoluta de lo sabido, en el caso de los saberes falibles nunca [se] estaría justificado en afirmar que alguien sabe, sino sólo que cree saber. Si queremos aplicar el concepto de 'saber' a saberes objetivamente justificados pero falibles, no podemos incluir en él la verdad absoluta de lo sabido" (1982:185).

Así, Villoro elimina del análisis clásico de saber la segunda condición sobre la verdad de una idea; y redefine la tercera, relacionada con la justificación que debe tener la persona para creer en esa idea, como "tener razones objetivamente suficientes para creer en ella" (1982:175.) Una razón es objetivamente suficiente para creer, con independencia del juicio de quien la sustenta, si es suficiente para cualquier sujeto posible 
de una comunidad epistémica pertinente; para lo cual se requiere el consenso de todos sus miembros. Comunidad epistémica pertinente es el conjunto de sujetos epistémicos pertinentes para una creencia - $\mathrm{y}$ éstos son todos los sujetos a los que les sean accesibles las mismas razones y no otras (1982:147.)

Este análisis contiene el segundo sentido de la noción de objetivo. Villoro afirma: “Objetividad no significa lo mismo que intersubjetividad pero implica intersubjetividad" (1993:345). En este sentido, la intersubjetividad es garantía de la verdad de un juicio, pues establece su validez con independencia de quien lo sustenta; es criterio de objetividad (1982:150). Las razones que nos permiten aceptar una creencia son "objetivas" en el sentido de que son incontrovertibles para cualquier sujeto de una comunidad epistémica; luego son intersubjetivas. Las razones objetivas en ese sentido justifican una creencia intersubjetiva (1993:345).

Olivé (2000) añade que la objetividad se refiere a la posibilidad de reconocimiento público en una comunidad de que hay una situación de hecho. Tal reconocimiento descansa en razones objetivamente suficientes, y por eso es racional. Por otra parte, la verdad de una proposición no es independiente de las razones de los sujetos para aceptarla, y por eso la verdad se identifica con aceptabilidad racional. La verdad de una proposición significa adecuación con la realidad; y no correspondencia con hechos objetivos, dados con independencia de los marcos conceptuales (Olivé 2000).

El constructivismo pluralista incorpora los conceptos epistémicos reformulados de objetividad, verdad y saber, y desarrolla la idea pluralista de racionalidad referente a la justificación dentro de una comunidad epistémica pertinente. Aplica un concepto de racionalidad instrumental, incluyendo racionalidad de fines y racionalidad de medios a fines, así como el concepto de racionalidad del desarrollo científico donde la epistemología señala las formas racionales de buscar el conocimiento. La racionalidad epistémica basada en las razones objetivamente suficientes guía la elección de creencias que constituyen genuino conocimiento la objetividad presupone la racionalidad (Olivé 2000).

La pluralidad es un rasgo constitutivo de la ciencia: lo que para una comunidad es saber objetivo puede no serlo para otra, relativizándose así el saber objetivo a las comunidades epistémicas y sus recursos (Olivé 2000); entonces la investigación en diferentes comunidades bajo marcos conceptuales inconmensurables puede conducir a un conocimiento legítimo de la realidad. Esto permite ver que las comunidades científicas del pasado han tenido genuino conocimiento de la realidad al igual que las actuales, incluso aunque en el futuro llegaran a abandonarse sus teorías (Olivé1999).

\section{Un análisis de ambas posturas metodológicas en la investigación en las disciplinas sociales}

Ambos enfoques parten de la concepción de la naturaleza de los hechos y fenómenos sociales por el investigador, ya sea buscando establecer intersubjetivamente regularidades causales, o rechazando la pretensión de que puedan generalizarse tales relaciones; y en consecuencia, de los objetivos y métodos, de la actitud hacia los valores epistémicos, y de los modelos del conocimiento, cientificidad y racionalidad que adoptará.

a. Los objetivos de la investigación. Las ciencias naturales se abocan a explicar y predecir el comportamiento de los fenómenos de la naturaleza al generalizar y sustanciar empíricamente las leyes y teorías que describen y explican las regularidades que éstos exhiben, y que son consensuadas como conocimiento por las comunidades científicas. 
En las disciplinas sociales bajo el monismo metodológico, el objetivo de la investigación es explicar las regularidades que exhiben los fenómenos humanos mediante metodologías consensuadas basadas en análisis cuantitativos con rigor metodológico, para establecer sus resultados replicables como hechos intersubjetivos, e inferir conclusiones generalizables con implicaciones para la teoría que sean aceptables intersubjetivamente como conocimiento en las comunidades de investigación.

Y bajo el dualismo metodológico, los objetivos son comprender e interpretar los hechos y fenómenos sociales, para conocerlos en toda su riqueza, profundidad y complejidad, y describirlos casuística y subjetivamente desde los marcos conceptuales y modelos analíticos cualitativos.

b. Los valores epistémicos. En las ciencias de la naturaleza la investigación se caracteriza por verdad, intersubjetividad, replicabilidad y precisión. Desde un instrumentalismo anti realista, la verdad de las teorías no se encuentra en la adecuación de su ontología con la realidad, sino en la adecuación de sus predicciones con el comportamiento de los fenómenos; siendo por lo tanto empíricamente adecuadas esas teorías.

En la investigación cuantitativa en las disciplinas sociales, la intersubjetividad caracteriza a las metodologías, así como la verdad, la intersubjetividad y la replicabilidad caracterizan a sus resultados: verdad, como la adecuación ex post de los resultados de los modelos con el comportamiento de los fenómenos, la cual es válida en las comunidades de investigación; intersubjetividad, al establecerse hechos mediante metodologías convencionales que eliminan el sesgo personal del investigador -intersubjetividad que es matizada al replicarse el proyecto- y replicabilidad, al cambiarse supuestos, metodologías o las muestras originales en proyectos subsiguientes con las mismas hipótesis, y obtenerse resultados consistentes.

Asimismo, quienes se adhieren a las tesis del dualismo metodológico dirigen la investigación a conocer sus objetos de estudio de manera directa, individual y subjetiva, buscando determinar la verdad de sus conclusiones como su adecuación con éstos mediante juicios basados en un criterio personal, sin pretender establecer un valor intersubjetivo para sus observaciones, argumentos y conclusiones.

c. Las regularidades en el comportamiento de los fenómenos. La ciencia busca aprehender las regularidades que se observan en la naturaleza, tales como las constantes universales y las leyes que asocian necesariamente a un efecto con sus causas.

Por su parte, la investigación cuantitativa busca explicar las regularidades observadas en los eventos de la naturaleza humana estableciendo sus determinantes con altos niveles de confianza estadística, para generalizar como conocimiento esas funciones explicativas al consensuarse en una comunidad epistémica la aceptabilidad racional de sus resultados de investigación por su adecuación con la evidencia empírica; esto es, a partir de razones objetivamente -es decir, intersubjetivamente- suficientes. $Y$ las predicciones probabilísticas de tales funciones explicativas juegan un papel central en la toma de decisiones, así como en el análisis y trazado de políticas en economía y administración.

d. El carácter epistémico de las teorías y generalizaciones empíricas. Desde la epistemología tradicional, teorías y leyes científicas pueden considerarse como conjeturas falsables.

Pero en las disciplinas sociales, las relaciones funcionales generalizables sobre regularidades empíricas establecidas desde las teorías generales revisten un carácter esencialmente probabilístico (15); y predicciones que no son precisas no pueden contraponerse con la evidencia ni ser falsadas, subsistiendo así 
inevitablemente un pluralismo teórico aun en áreas donde se han desarrollado metodologías cuantitativas para ir más allá de la subjetividad en la investigación, y establecer hechos y conclusiones intersubjetivos tales como el conductismo, la economía matemática o la administración financiera. Las funciones explicativas establecidas no son generalizaciones empíricas con carácter de conjeturas falsables, sino herramientas clave para intervenir y moldear el entorno social, así como el comportamiento de individuos, grupos, organizaciones y sociedades.

e. Las disciplinas sociales y el convencionalismo de Lakatos. Los criterios de cientificidad y racionalidad de Lakatos se aplican únicamente a las ciencias naturales (16). Por otra parte, para Kuhn (1964) el desarrollo de la ciencia eventualmente alcanza un punto en el que las comunidades de investigación de un campo del conocimiento, que hasta entonces habían trabajado bajo los estándares y metodologías de una de las teorías alternativas coexistentes en un mismo momento, alcanzan un consenso sobre una de ellas reconociéndola como el primer paradigma universalmente aceptado en la disciplina, en lo que constituye su transición a la etapa paradigmática o de ciencia normal.

Dejar atrás la etapa pre-paradigmática con desacuerdos sobre aspectos fundamentales de la disciplina y con diferentes teorías alternativas, es un cambio cualitativo crucial ya que la adquisición de un paradigma y del tipo más esotérico de investigación que permite es un signo de madurez en el desarrollo de cualquier campo científico. En realidad, es difícil encontrar otro criterio que tan claramente proclame como ciencia a un campo, que el que se establezca un paradigma que muestre ser capaz de guiar la investigación de todo el grupo.

En estos términos, las disciplinas sociales permanecen aún en una etapa pre-paradigmática, pre-científica de desarrollo, en la que no ha sido aceptada una teoría por las comunidades de investigación de alguna disciplina como su primer paradigma; y por lo tanto, no muestran revoluciones científicas ni series de teorías, el cual es el patrón de desarrollo de las ciencias que han alcanzado su madurez.

Sin embargo, Kuhn (1964) concluye una relación de las transiciones a la etapa paradigmática de las ciencias naturales señalando que tales transiciones podrían estar ocurriendo ahora en partes de las ciencias sociales [sic]; pero que es controvertido aún señalar qué partes de las mismas han adquirido ya tales paradigmas. Aquí es importante destacar que el orden temporal en el que maduraron tales ciencias está en función de la complejidad de los fenómenos que estudian. La investigación en las disciplinas sociales involucra la descripción y explicación del comportamiento humano individual o colectivo, incluso en distintos contextos institucionales, exhibiendo claramente los fenómenos de la mayor complejidad observable -lo que también explica el inicio relativamente reciente de su estudio formal.

f. Las disciplinas sociales y el constructivismo pluralista. Buscando superar el escepticismo, los planteamientos de Olivé reivindican el pluralismo en la ciencia, sustituyendo el ideal de una racionalidad universal por el de racionalidad en una comunidad epistémica. Su concepción de objetividad como aceptabilidad racional en condiciones realmente existentes para tal comunidad determina que es racional aceptar la existencia de objetos por razones incontrovertibles, y que representa genuino conocimiento. EI constructivismo pluralista asocia las nociones de verdad y justificación y afirma que verdad es aceptabilidad racional de una creencia dadas ciertas razones en un esquema conceptual; pero una proposición no puede ser verdadera con respecto de uno de ellos y falsa con respecto de otro (Olivé 1999). 
Lo anterior legitima el que las comunidades académicas consideren como conocimiento genuino resultados de investigación que encuentren aceptabilidad racional en una o en varias, pero no en todas las comunidades epistémicas; lo cual es el caso con los resultados de investigación cuantitativa en las disciplinas sociales. Sin embargo, estos conceptos no se aplican a los resultados de investigación cualitativa en las disciplinas sociales bajo la visión del dualismo metodológico, toda vez que los mismos parten de inferencias personales y juicios subjetivos, y no buscan establecer un consenso dentro de una comunidad de investigación.

\section{Consideraciones finales y conclusiones}

La controversia en las disciplinas sociales en torno a los objetivos, métodos y criterios de validez de resultados y conclusiones de investigación parte de dos posturas con respecto a la concepción de la naturaleza de los hechos y fenómenos humanos; que a su vez determinan losmodelos del conocimiento, cientificidad y racionalidad que se considerarán adecuados. La investigación cuantitativa del naturalismo busca establecer como saber su explicación generalizable de ellos; mientras que, bajo su propósito de conocer los fenómenos sociales en toda su complejidad y riqueza, los resultados de índole descriptiva y casuística de la investigación cualitativa se presentan como opiniones expertas basadas en la autoridad personal y prestigio del investigador.

Subsiste un pluralismo teórico en el nivel de las teorías generales de las disciplinas sociales, y aún en el de sus especialidades con un alto grado de desarrollo de la investigación cuantitativa, donde las funciones explicativas permiten hacer simulaciones y generar predicciones valiosas para intervenir y moldear el entorno social. Tales generalizaciones empíricas ciertamente no son falsables, pero cuando nuevas concepciones, supuestos o técnicas cuantitativas producen mejores resultados predictivos, tales modelos son sustituidos por los de una nueva generación.

Sin embargo, está también el caso de teorías que describen y prescriben de manera específica el comportamiento de fenómenos particulares, y que son falsables y científicas de acuerdo a las consideraciones antecedentes. Éste es el caso, por ejemplo en finanzas, de las teorías de la caminata aleatoria y de los mercados eficientes (17); o en economía, del nuevo paradigma o nueva economía, en boga en la última década del siglo pasado. La predicción de esta última teoría de que el ciclo económico sería controlado permanentemente a través de las medidas de política económica fue falsada por la recesión de los primeros años de este siglo. Pero la investigación sobre ambas teorías financieras se caracteriza por producir evidencia conflictiva: la corroboración de sus tesis va en paralelo con evidencia empírica de ejemplos en contrario pertinentes (18).

Durante largo tiempo, la naturaleza probabilística de los fenómenos y las limitaciones de los modelos explicativos de las disciplinas sociales han puesto en entredicho lo apropiado del enfoque cuantitativo para la investigación de los fenómenos de la naturaleza humana. Entonces, es importante observar con respecto a las generalizaciones empíricas que en las ciencias de la naturaleza hay una creciente incredulidad sobre su universalidad e invariancia, lo cual delinea un mundo en el que la causalidad y la regularidad están estructuradas de manera mucho más compleja, y que se revelan en patrones menos obvios de lo que tradicionalmente se ha creído; pero sugiere también que el objetivo de discernir regularidades en los hechos y en los fenómenos sociales y explicarlas cuantitativamente no resulta implausible. Ciertamente, científicos y filósofos de la ciencia consideran que la comprensión científica requiere de enunciados empíricos 
generales aun cuando éstos no satisfagan todos los criterios tradicionalmente atribuidos a las leyes; y las leyes probabilísticas ocupan un lugar prominente incluso en las ciencias de la naturaleza.

El constructivismo pluralista de Olivé no puede extenderse para buscar legitimar el pluralismo teórico de las disciplinas sociales. Sus planteamientos legitiman el que las comunidades epistémicas consideren como conocimiento genuino proposiciones y teorías que encuentren aceptabilidad racional en una o en varias comunidades, aunque no en todas ellas; que lo que es conocimiento objetivo para una comunidad epistémica no lo sea para otra (Olivé 1999, 2000); y que la investigación en diferentes comunidades bajo esquemas conceptuales inconmensurables pueda conducir a un conocimiento legítimo de la realidad.

Pero la concepción de Kuhn sobre la pluralidad sincrónica en la ciencia, cuando diferentes comunidades en un campo del conocimiento se encuentran estudiando los mismos fenómenos bajo teorías rivales, es que tal situación significa que esa disciplina permanece en una etapa pre-científica de evolución, "en su prehistoria como ciencia" (1964:21), donde en ausencia de un paradigma o de algún candidato a paradigma, la investigación es mucho más una actividad casi aleatoria que la que hace familiar el desarrollo científico subsecuente (1964:15); y que superar esa condición pre-paradigmática permite a la disciplina alcanzar su madurez y el status de ciencia.

En las disciplinas sociales, la investigación se caracteriza actualmente por una situación pre-paradigmática con distintas líneas de investigación que adoptan diferentes objetivos, supuestos, metodologías y marcos conceptuales que no convergen en un único programa al carecerse de la guía unificadora de un paradigma establecido; y Lakatos (1970) exhibe un intenso desprecio hacia una investigación con estas particularidades. Desde luego, tal actitud muestra que no él alcanza a comprender:

a) Que cada una de las ciencias naturales y cada una de sus especialidades ha pasado a la etapa paradigmática necesariamente desde la etapa pre-paradigmática, en la cual se desarrollaban los trabajos a partir de los cuales eventualmente surgiría aquel primer paradigma que finalmente se implantaría a lo largo de toda la disciplina, unificando entonces ya a todos los puntos de vista. Claramente tal primer paradigma no habría surgido simplemente de la nada, sino que la investigación que se llevaba a cabo en la etapa precientífica exploraba en su profundidad y complejidad los fenómenos propios de su campo de estudio, y redondeaba cada vez de mejor manera las posibilidades de alcanzar una comprensión unificadora en la disciplina.

b) Que en el estudio de la historia de la ciencia natural se perfila un patrón que sugiere que el orden en el que las ciencias naturales e incluso varias de sus especialidades alcanzaron su madurez está en función del creciente grado de complejidad que caracteriza a los fenómenos sobre los que trabaja cada uno de esos campos del conocimiento; a la correspondiente decreciente claridad con que se delinean y se revelan al observador esos eventos, pudiendo él discernir sus regularidades; y a la consecuente progresiva dificultad para investigar y establecer empíricamente tales leyes científicas y las teorías que las describan y expliquen.

Al respecto, Kuhn (1964: prefacio y caps. I y II) señala los periodos en que ha ocurrido esta transición en diversas ciencias o en sus especialidades. Así, desde la antigüedad se estableció en la astronomía su primer paradigma; y entre la Edad Media y el siglo XVIII, vivieron tal momento en su evolución algunas áreas de la física, tales como la dinámica, la óptica y la electricidad, en este mismo orden -y la mayor parte del resto de ellas, lo tuvieron en el XIX. En la química tales eventos comenzaron a tener lugar a partir del siglo XVII; y las 
distintas partes de la biología 'cruzaron la frontera entre lo que el historiador podría llamar su prehistoria como ciencia y su historia propiamente dicha,' durante los siglos XVIII y XIX.

Así, este esquema abarca desde las regularidades más obvias y sencillas de observar como lo son las de la mecánica celeste, hasta las de la biología, que incorpora ya la vida a las dimensiones físico-químicas de sus objetos de estudio. Claramente, la tarea de las disciplinas sociales, que involucra la exploración de la conducta humana en su manifestación tanto individual como grupal -e incluso asociando a ella los aspectos abstractos de elementos institucionales de distintos tipos y en diversos contextos- muestra el mayor grado de complejidad entre los campos del conocimiento (Salas 2008).

En conclusión, las propuestas filosóficas dejan abierta la controversia entre ambas posturas metodológicas sobre las concepciones y enfoques apropiados para explorar los fenómenos sociales. Las limitaciones de las leyes de la naturaleza muestran la plausibilidad de modelar las regularidades de los fenómenos sociales, pero este tipo de investigación enfrenta las formidables debilidades de la estrechez de los ámbitos del conocimiento en los que ha podido ser plenamente aplicada, así como de la exclusividad de los espacios institucionales en donde la práctica profesional requiere de tales modelos cuantitativos -la cual no sería extrapolable a otras disciplinas, o siquiera a otras especialidades de la administración o de la economía mismas.

Los planteamientos del convencionalismo de Lakatos restituyen los criterios de demarcación y racionalidad sólo a las ciencias de la naturaleza; mientras que los del constructivismo pluralista establecen una racionalidad para resultados y conclusiones de la investigación cuantitativa, y los justifican como genuino conocimiento. Pero sus tesis no legitiman el pluralismo teórico prevaleciente en estas disciplinas, ya que se contraponen a la explicación del desarrollo y progreso de la ciencia por una teoría ampliamente aceptada.

\section{Notas}

(1) Una versión preliminar de este texto aparece con el título "El conocimiento, los objetivos y los métodos de investigación en las disciplinas sociales" en la Memoria del XV Congreso Internacional de Contaduría, Administración e Informática, FCA-ANFECA, UNAM, México, 2010, pp. 1-27.

(2) La elección inevitablemente arbitraria de diseños de investigación, modelos, técnicas cuantitativas, supuestos y decisiones metodológicas trasciende la subjetividad del investigador al ser consensuada en las comunidades de investigación, dando validez intersubjetiva a resultados, conclusiones e implicaciones del proyecto para la teoría.

(3) Igualmente, para Moritz Schlick, fundador del Círculo de Viena, "toda cognición en cualquier dominio del conocimiento es esencialmente establecer una correspondencia entre los hechos del mundo y un sistema de símbolos" -en contraposición a los sistemas de filosofía para los cuales "cognición significaba encontrar una verdad oculta detrás de las apariencias, que podía ser descubierta por el poder de la razón" atribuido al filósofo calificado (Frank 1950:29).

(4) Kuhn Ilama ciencia normal a la actividad científica realizada bajo los supuestos y metodología de un paradigma mientras está vigente, y que se contrapone a la ciencia extraordinaria que tiene lugar durante la transición de un paradigma a otro en una revolución científica. Un paradigma es un caso ejemplar de investigación científica que contiene leyes y teorías, sus aplicaciones a la solución de problemas y las técnicas matemáticas y experimentales aplicadas. Tales logros, universalmente reconocidos, proporcionan temporalmente a los científicos problemas y soluciones modelo. Los paradigmas son aceptados por consenso en las comunidades científicas al implantarse en una disciplina su primer paradigma, o al sustituirse en una revolución científica el paradigma vigente por otro que resuelve las anomalías que habían cuestionado la validez de aquél. Y una anomalía consiste en una discordancia persistente entre las 
expectativas generadas bajo un paradigma con relación a la forma en que se presupone que se manifestará un fenómeno y el comportamiento con el que el mismo se observa verdaderamente en la naturaleza.

(5) A principios del siglo XX, Pierre Duhem (1993) advierte que una experiencia de Física no sólo es observar un fenómeno; es además su interpretación teórica. Asimismo, dentro del Círculo de Viena, Otto Neurath sustenta ya el carácter convencional y corregible del lenguaje observacional (Velasco 2000).

(6) Michael Polanyi (1958) señala ya con anterioridad que ningún esquema 'objetivista' puede dar cuenta de la aceptación o rechazo de teorías por el científico.

(7) Richard Braithwaite coincide en que exceptuando las generalizaciones de hechos simples observables (por ejemplo, todos los cisnes son blancos), no es más posible la refutación completa que la prueba completa.

(8) Para Bertrand Russell, la ciencia en ningún momento está totalmente en lo cierto, pero rara vez está totalmente equivocada; y tiene en general mayores posibilidades de estar en lo cierto que las teorías no científicas.

(9) A fines del siglo XIX y principios del XX, Henri Poincaré, Gaston Milhaud y Edouard Le Roy explican la actividad científica como un convencionalismo basado en decisiones metodológicas, no en pruebas empíricas.

(10) Larry Laudan (1977, 1981) concibe el progreso científico dentro de tradiciones de investigación análogas a paradigmas y a programas de investigación- con supuestos metafísicos y elementos metodológicos, que norman la generación, evaluación y sustitución de teorías, en términos del progreso o éxito predictivo.

(11) En esta misma línea de pensamiento, a fines del siglo XIX surge la hermenéutica como modelo científico alternativo, específico para guiar y justificar la investigación en las disciplinas sociales (Dilthey, Rickert, Gadamer, Habermas, Velasco); pero ha recibido poca atención de las comunidades de investigación.

(12) Entre quienes destacan Henri Poincaré, Gaston Milhaud, Edouard Le Roy, Pierre Duhem, Alexandre Koyré, Edwin Burtt, William Whewell, Ludwick Fleck y Michael Polanyi, cuyas ideas antecedieron a las de Kuhn.

(13) La inconmensurabilidad de paradigmas es la imposibilidad de que establezcan pleno contacto con el punto de vista ajeno, comunidades científicas basadas en distintos paradigmas. La comunicación es inevitablemente parcial entre ellas debido a desacuerdos sobre sus propósitos, estándares, definiciones de ciencia y problemas a resolver, entre otros; así como a una ontología asociada al paradigma, que dice cómo es el mundo, qué entidades existen y qué conducta les está permitida. "Los defensores de paradigmas rivales ejercen su oficio en mundos diferentes, y ven cosas diferentes al ver en la misma dirección" (Kuhn 1964: caps. IV y XII.)

(14) Ana Pérez (1999:211) pregunta, ¿qué queda de la idea de que hay algo 'ahí afuera' que es independiente de la mente o del lenguaje? Génelot (1998) afirma: el paradigma de la complejidad nos enseña que no hay una realidad objetiva, allí afuera, independiente de los integrantes, con el mismo sentido para todos, hablar de complejidad es reconocer que existen múltiples versiones de la realidad, que influyen en las acciones de los participantes.

(15) Se visualiza a un valor que predice un modelo cuantitativo como la media de una distribución normal de probabilidad que permite determinar distintos rangos, dentro de cada uno de los cuales podrá esperar observarse con una probabilidad dada el verdadero valor que mostrará cierta variable al ocurrir un fenómeno. Por ejemplo, en la relación funcional inversa entre precios y cantidades, se espera que a una reducción en precios corresponda un aumento en el volumen de ventas; sin embargo -y suponiendo una función de demanda con una elasticidad unitaria en ese punto con respecto a precios-, a una rebaja quizá de un $20 \%$, no se espera que siga un aumento en ventas sino de alrededor de un $20 \%$. Toda vez que ésta y cualquier otra función son estimadas cuantitativamente a partir de observaciones que muestran desviaciones contra los valores que predice la curva, las bandas de confianza estadística de $95 \%$ y $99 \%$ para una predicción a partir de la misma estarán delimitadas aproximadamente dentro de dos y tres errores estándar, respectivamente, a ambos lados de la media de la distribución normal de probabilidad correspondiente -media que es representada por la curva misma. Pero sobre todo, cabe recordar que los 
extremos de esta curva son asintóticos sobre el eje horizontal; es decir, que no es determinable un rango específico dentro del cual podrá encontrarse el 100\% de probabilidad para una predicción.

(16) De hecho, Lakatos (1970) desprecia vivamente la investigación en las disciplinas sociales tal como se lleva a cabo actualmente.

(17) Louis Bachelier (1900) desarrolló la teoría matemática de los procesos aleatorios, observando que en los mercados de valores los precios siguen un comportamiento al azar, que sería impredecible por un especulador. Y para Eugene Fama (1970), en los mercados eficientes los precios reflejan toda la información existente, por lo que ningún inversionista podría hacer consistentemente pronósticos superiores de precios de activos financieros -en un mercado eficiente el valor esperado de la diferencia entre el precio en el mercado y el precio esperado por los inversionistas es igual a cero, dado un conjunto particular de información. Así, ambas teorías serían falsadas si un inversionista obtuviera consistentemente rendimientos extraordinarios en un mercado financiero.

(18) Cabello et al. (2004) encuentran que el mercado del peso mexicano y el dólar americano no es eficiente; y Díaz (2005) muestra que pueden obtenerse rendimientos extraordinarios en el mercado bursátil mexicano durante periodos largos.

\section{Bibliografía}

Allchin, D. 2007. Teaching science lawlessly. En: P. Heering y D. Osewold (eds.). Constructing scientific understanding through contextual teaching. Berlin: Frank \& Timme, pp.13-31.

Ayer, A. 1956. Language, truth and logic. London: Victor Gollancz Ltd.

Bachelier, L. 1900. Théorie de la speculation. Paris: Ann. Sci. Ecole Norm. Sup. (3) No. 1018.

Barnett, L. 1974. The universe and Dr. Einstein. New York: Bantam Books.

Boyd, R. 1992. Constructivism, realism and philosophical method. En: J. Earman (comp.). Inference, explanation and other frustrations. Berkeley: University of California Press, pp. 131-198.

Cabello, A., Ortiz, E. y Johnson, R. 2004. Eficiencia del mercado y desequilibrios a largo plazo en la paridad del poder adquisitivo del peso mexicano bajo distintos regímenes cambiarios.Memoria del IX Foro de Investigación. México: FCA-ANFECA, UNAM.

Carnap, R. 1961. La superación de la metafísica por medio del análisis lógico del lenguaje. México: Cent. Est. Fil., UNAM. Colección Cuadernos, No. 10.

Daston, L. y Galison, P. 2007. Objectivity. New York: Zone Books.

Díaz, A. 2005. Un sistema computarizado para análisis de acciones bursátiles en México. Tesis doctoral no publicada. México: DCA, UNAM.

Duhem, P. 1993. La théorie physique. Son objet - sa structure. Paris: Lib. Phil. J. Vrin.

Etkin, J. 1996. La empresa competitiva. Grandeza y decadencia. Santiago: McGraw Hill.

Etkin, J. y Schvarstein, L. 1995. Identidad de las organizaciones, invariancia y cambio. Buenos Aires: Paidós.

Fama, E. 1970. Efficient capital markets: A review of theory and empirical work. Journal of Finance 25: 383417. 
Fleck, L. 1986. La genesis y el desarrollo de un hecho científico. Madrid: Alianza.

Frank, P. 1950. Modern science and its philosophy. Cambridge: Harvard University Press.

Génelot, D. 1998. Manager dans la complexité. Paris: INSEP Éditions.

Hempel, C. 1965. Problemas y cambios en el criterio empirista de significado. En: A. Ayer (comp.). El positivismo lógico. México: FCE, pp. 115-136.

Knight, D. 1992. Ideas in chemistry. A history of the science. London: The Athlone Press Ltd.

Kuhn, T. 1964. The structure of scientific revolutions. Phoenix: University of Chicago Press.

Kuhn, T. 1977. The essential tension. Phoenix: University of Chicago Press.

Lakatos, I. 1970. Falsification and the methodology of scientific research programmes. En: I. Lakatos y A. Musgrave (eds.). Criticism and the growth of knowledge. Cambridge: Cambridge University Press, pp. 91-195.

Laudan, L. 1977. Progress and its problems. Berkeley: California University Press.

Laudan, L. 1981. A problem-solving approach to scientific progress. En: I. Hacking (comp.). Scientific revolutions. Oxford: Oxford University Press, pp. 144-155.

Morin, E. 1996. Introducción al pensamiento complejo. Barcelona: Gedisa.

Nozick, R. 2008. Knowledge and skepticism. En: E. Sosa, et al. (eds.). Epistemology. An anthology. Malden, MA: Blackwell Publishing, pp. 255-279.

Olivé, L. 1999. Multiculturalismo y pluralismo. México: Paidós.

Olivé, L. 2000. El bien, el mal y la razón. Facetas de la ciencia y de la tecnología. México: Paidós.

Pérez, A. 1999. Kuhn y el cambio científico. México: FCE.

Polanyi, M. 1958. Personal knowledge: Towards a post-critical philosophy. London: Routledge and Kegan Paul.

Pojman, L. 2001. What can we know? An introduction to the theory of knowledge. Belmont, CA: Wadsworth Thomson Learning.

Popper, K. 1969. Logik der Forschung. Tübingen: J.C.B. Mohr.

Popper, K. 1979. Tres concepciones sobre el conocimiento humano. En: K. Popper, El desarrollo del conocimiento científico: Conjeturas y refutaciones. Buenos Aires: Paidós, pp. 116-141.

Putnam, H. 1981. Reason, truth and history. Cambridge: Cambridge University Press.

Putnam, H. 1987. The many faces of realism. La Salle, Illinois: Open Court. 
Rendón, M. y Montaño, L. 2004. Las aproximaciones organizacionales. Caracterización, objeto y problemática. Contaduría y Administración 213: 101-120.

Russell, B. 1969. The ABC of relativity. New York: The New American Library, Mentor Books.

Salas, H. 2008. Reflexiones sobre la presencia de regularidades en los fenómenos sociales y sobre la posibilidad de investigarlas desde un monismo metodológico. Contaduría y Administración 224: 161-182.

Schlick, M. 1965. Positivismo y realismo. En: A. Ayer (comp.). El positivismo lógico. México: FCE, pp. 88-114.

Toulmin, S. 1960. The Philosophy of science. New York: Harper and Row.

Velasco, A. 1995a. Filosofía de la ciencia, hermenéutica y ciencias sociales. Ciencia y desarrollo 21(125): 6981.

Velasco, A. 1995b. La hermeneutización de la filosofía de la ciencia contemporánea. Diánoia/ Anuario de filosofía 61: 53-64.

Velasco, A. 2000. Tradiciones naturalistas y hermenéuticas en la filosofía de las ciencias sociales. México: UNAM-Campus Acatlán.

Villoro, L. 1982. Creer, saber, conocer. México: Siglo XXI.

Villoro, L. 1993. Respuesta a discrepancias y objeciones. En: E. Garzón y F. Salmerón (comps.). Epistemología y cultura. En torno a la obra de Luis Villoro. México: UNAM, pp. 331-350.

Waters, K. 1998. Causal regularities in the biological world of contingent distributions. Biology and Philosophy 13: 5-36.

Recibido el 11 Ago 2010

Aceptado el 3 Ene 2011 